\title{
Effects of analgesic \& Anti-inflammatory drugs on orthodontic tooth movement- A biochemical \& Histological study in guinea pigs
}

\author{
Dr.Suchita Tarvade/ Daokar1, Dr.Sadashiv Daokar ${ }^{2}$, Dr. P.V. Hazarey. ${ }^{3}$ \\ 1-Professor \& P.G.Guide, Dept.of Ortho, C.S.M.S.S. Dental College, Aurangabad. \\ 2- Professor \& P. G. Guide, Dept. of conservative dentistry, C.S.M.S.S. Dental College, Aurangabad. \\ 3- Professor, H.O.D.,\& P.G.Guide, Dept.of Ortho, S.P.D.C. Dental College,Nagpur.
}

\section{Introduction :-}

The essence of orthodontic treatment is the movement of teeth throughbone to obtain a more perfect dental occlusion. Mechanical forces exerted ontooth and transmitted to the surrounding tissues of periodontal ligament initiates the remodeling activity and facilitates the movement of teeth through bone.

Cells of the nervous, immune and endocrine systems become involved in the activation and response of periodontal ligament and alveolar bone cells during tooth movement.Orthodontic tooth movement has an inflammatory response and so evokes painwhich is the most unpleasant symptom which drives the patient to seek medicaments. Analgesic drugs are commonly prescribed in day to day orthodontic practice to control pain evoked by orthodontic forces. These drugs are also available as OTC ( over-the -counter) drugs and can be bought from medical shops without prescription.

$\mathrm{T}$ he present investigation is done to study the effects of three different analgesic and anti-inflammatory drugs, acetaminophen, ibuprofen and nimesulide, on the rate of orthodontic tooth movement in guinea pigs.

\section{Aims and Objectives:-}

1. Compare the effects of three commonly used analgesics acetaminophen. Ibuprofen and nimesulide on the rate of orthodontic tooth movement in male guinea pigs for 72 hours.

2. Compare the effects of three commonly used analgesics acetaminophen. Ibuprofen and nimesulide on the acid phosphatase level in serum during orthodontic tooth movement.

3. To study and compare the histological changes in periodontal ligament during orthodontic tooth movement with the administration of acetaminophen, ibuprofen and nimesulide.

4. To correlate the histological changes with biochemical changes during orthodontic tooth movement when these analgesic drugs are administered.

\section{Review of literature :-}

In 1880, Norman Kingsley, discussed about alveolar bone and its response to forces . He explained orthodontic movement of teeth as a result of elasticity of the alveolar bone.

In 1888, Farrar described the orthodontic movement of teeth as a result of the resorption and apposition of bone and bending of the alveolar process.

Herzberg in 1932 was the first to move a human tooth with an orthodontic appliance and study its surrounding tissues.

In 1953, Storey recognized 4 zones of activity around a tooth which was being moved with light orthodontic forces. On the pressure side resorption and then deposition, on the tension side deposition and then resorption.

D.L. Buck and D. H. Church in 1972 studied histological changes during human tooth movement.

Klein and Raisz in 1970 reported for the first time that prostaglandin stimulate bone resorption by acting on osteoclasts in tissue cultures.

Harell et al in 1977 suggested for the first time that prostaglandins may be important mediators of mechanical stress.

Storey in 1978 showed that inflammatory reactions are associated with the application of orthodontic forces.

Davidovitch and Shanfeld in 1980 also reported the involvement of PGE2 in bone remodeling of orthodontically treated cats showing the rise of PGE2 levels in alveolar bone.

Yamasaki in 1984 studied the effect of locally administered PGE upon tooth movement and showed increase rate in tooth movement. 
II. Materials and Methods:-

A total number of 28 healthy male guinea pigs were used in this study. The animals were divided into two main groups

1. Group I- 24 animals were used in this group for biochemical study.

2. Group II- 4 animals were used in this group for histological study.

Group I and Group II were further divided into 4 subgroups each

Subgroup I and II (a)- Animals in these subgroups were used as control . orthodontic treatment was given but no drugs were administered throughout the study to these animals.

Subgroup I \& II (b)- animals in these subgroups were given orthodontic treatment and acetaminophen suspension was administered 12 hourly for 3 days.

Subgroup I \& II ( c) - animals in these subgroups were given ibuprofen suspension 12 hourly for 3 days along with orthodontic treatment.

Subgroup I \& II (d) - animals included in these subgroups were administered nimesulide suspension 12 hourly for 3 days along with orthodontic treatment.

\section{Orthodontic appliance :-}

After sedating the guinea pigs with $2 \mathrm{mg} / \mathrm{kg}$ of ketamine intramuscularly bands with vertically welded eyelet were cemented on both mandibular central incisor.

A spring was prepared of 0.014 " (A. J. Wilcock's wire) with two vertical loops mesial arm slightly shorter then the distal arm. 2 helical coils of $1.5 \mathrm{~mm}$ inner diameter and one and a half turn were incorporated in these vertical loops in between the mesial and distal arm.

The spring was engaged in the two eyelets.

The guinea pigs were then isolated.They were given green leafy vegetables and were administered drugs .

The tooth separation measurements were done between the mesial margins of the incisal edges of the two mandibular incisors using vernier caliper. The recording were done prior to placement of the appliance, $24 \mathrm{hrs}$, 48 hours and 72 hours after orthodontic appliance placement. At each seating, measurement was repeated for 3 times, of each animaland a mean of 3 measurements was taken.

Fig 1:-

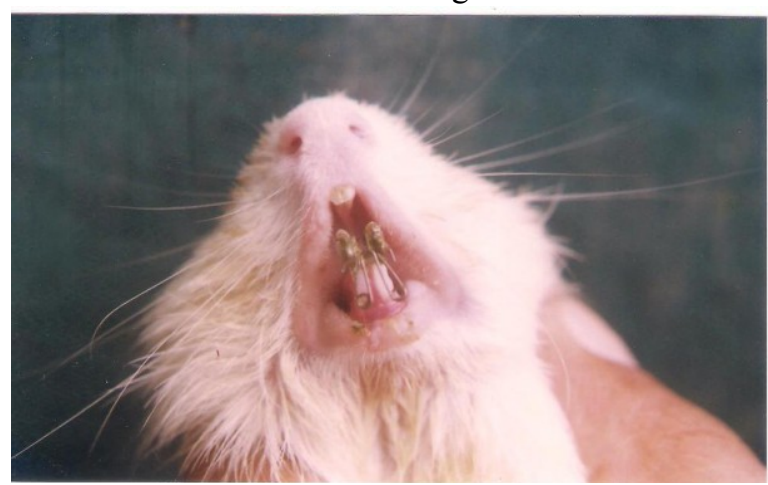

Blood was collected after 72 hours of appliance placement.
Fig 2:-

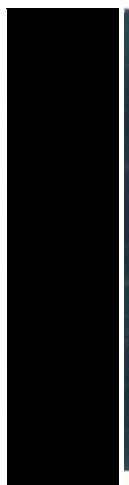

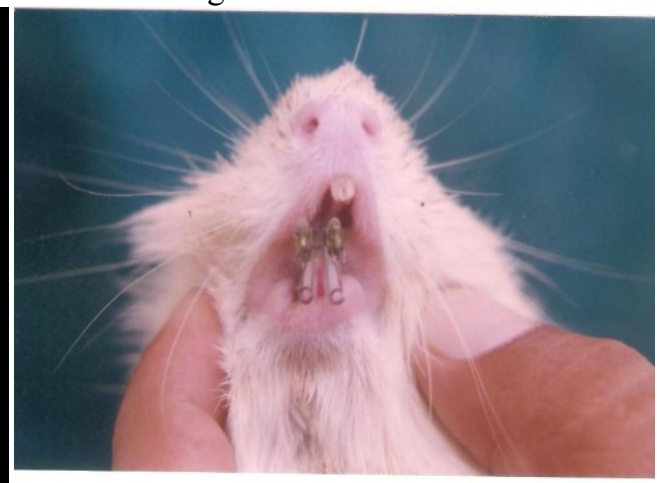

For histological examination, tissue was collected by administering a lethal dose of ketamine to all the animals of group II.

\section{Results:-}

Table no1:- the results of tooth separation in $\mathrm{mm}$ among the sub-groups were:

\begin{tabular}{|c|c|c|c|c|c|c|c|c|}
\hline \multirow[t]{2}{*}{ At } & \multicolumn{2}{|c|}{ Subgroup I (a) } & \multicolumn{2}{|c|}{ Subgroup I (b) } & \multicolumn{2}{|c|}{ Subgroup I (c) } & \multicolumn{2}{|c|}{ Subgroup I (d) } \\
\hline & Mean & S.D & Mean & S.D & Mean & S.D & Mean & S.D \\
\hline $24 \mathrm{hrs}$ & 1.56 & $+/ 0.12$ & 1.41 & $+/-0.14$ & 0.91 & $+/-0.11$ & 0.7 & $+/-0.104$ \\
\hline $48 \mathrm{hrs}$ & 2.66 & $+/-0.12$ & 2.53 & $+/-0.18$ & 1.4 & $+/-0.08$ & 1.25 & $+/-0.164$ \\
\hline $72 \mathrm{hrs}$ & 3.70 & $+/-0.08$ & 3.6 & $+/-0.17$ & 2 & $+/-0.08$ & 1.75 & $+/-0.148$ \\
\hline
\end{tabular}


Effects of analgesic \& Anti-inflammatory drugs on orthodontic tooth movement- A biochemical \&

Table no 2:- the results of Acid Phosphates level in the serum among the sub-groups were:-

\begin{tabular}{|c|c|c|c|c|c|c|c|c|}
\hline \multirow{2}{*}{ At } & \multicolumn{2}{|c|}{ Subgroup I (a) } & \multicolumn{2}{c|}{ Subgroup I (b) } & \multicolumn{2}{c|}{ Subgroup I (c) } & \multicolumn{2}{c|}{ Subgroup I (d) } \\
\cline { 2 - 9 } & Mean & S.D & Mean & S.D & Mean & S.D & \multirow{2}{*}{ Mean } & S.D \\
& & & & & & & & \\
\hline $24 \mathrm{hrs}$ & 2.05 & $+/-2.06$ & 1.81 & $+/-0.15$ & 0.81 & $+/-0.26$ & 0.86 & $+/-0.12$ \\
\hline $72 \mathrm{hrs}$ & 2.8 & $+/-2.06$ & 2.65 & $+/-0.15$ & 1.7 & $+/-0.08$ & 1.36 & $+/-0.20$ \\
\hline
\end{tabular}

The student ' $t$ ' test and ANOVA test were applied to all the observations .

Tooth separation and acid phosphatase level were tested for all the

Fig 3:-

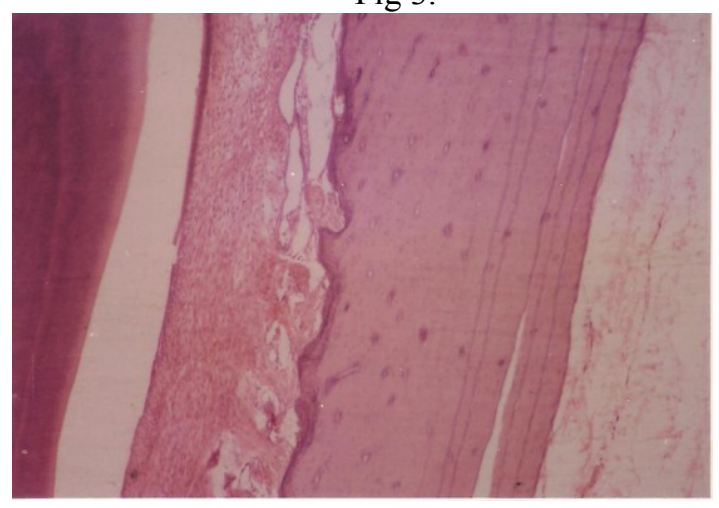

Fig 5:-

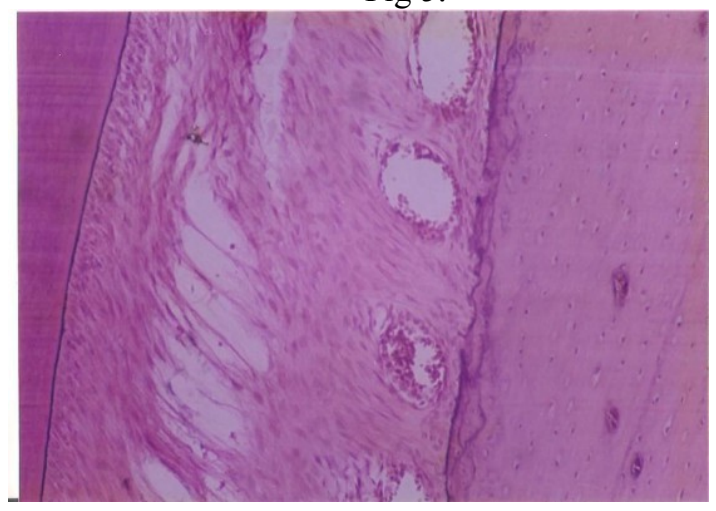

Fig 4:-

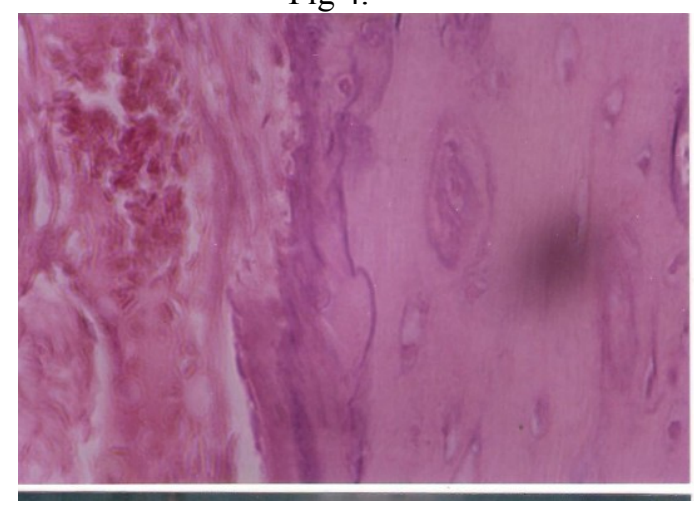

Fig 6:-

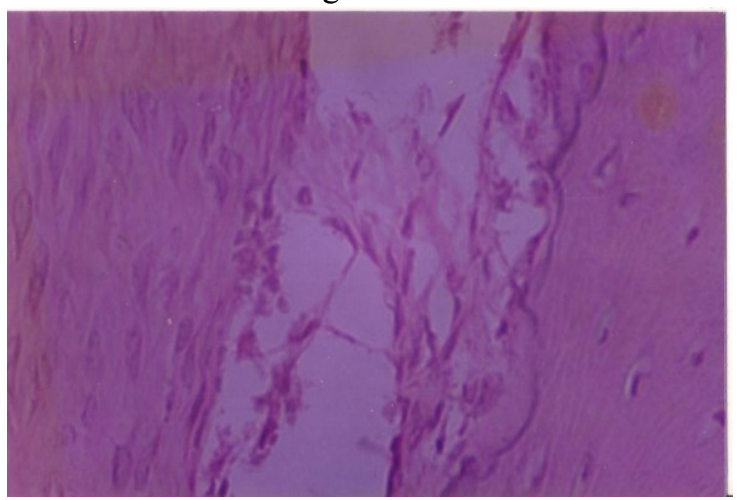

\section{Discussion :-}

Student ' $\mathrm{t}$ ' test and ANOVA test was performed to comoare the significance of difference between the tooth separation and acid phosphatase level in serum amongst the subgroups.

The above parameters were compared in between control group i.e; subgroup I(a) and

1. Subgroup I (b)- Acetaminophen

2. Subgroup I (c)- Ibuprofen

3. Subgroup I (d )- Nimesulide

When the parameters were compared between the 4 subgroups the acid phosphatase levels were found to be highest in control group followed by the acetaminophen subgroup. The acid phosphatase levels were found to be significantly lower in ibuprofen and nimesulide subgroup when compared to control group. When levels of acid phosphatase were compared within the subgroups again it was found that there was statistically significant. The histological finding showed serrated border of the bone and large no. of howship's lacunae and osteoclasts in the distal side of the tooth in control and acetaminophen subgroups, where as the ibuprofen and nimesulide subgroups showed fewer howship's lacunae and osteoclasts .

Thus the biochemical and histological findings were in correlation with each other.These findings were similar to the findings reported by Stephen Keeling et al. they investigated histochemically and biochemically acid and alkaline phosphatase level in serum and alveolar bone during orthodontic tooth movement in 288 male Sprague Dawley strain rats. 


\section{Conclusion :-}

In summary the mean tooth separation was found to be highest in control group (subgroup I a). This was followed almost the same amount of tooth separation in acetaminophen (subgroup I b). this suggest that acetaminophen can be administered to patients for pain control during orthodontic treatment.Minimal tooth separation was found in ibuprofen (subgroup I c) and nimesulide (subgroup Id). This suggest that ibuprofen and nimesulide decrease the rate of tooth movement and so should be avoided during orthodontic therapy.

Similar findings were found in acid phosphatase level in serum betweenthe subgroups suggesting that acid phosphatase level in serum reflect the alveolar bone turnover during orthodontic tooth movement.

Further these findings were also confirmed by light microscopic histological study.

The conclusions drawn from the study were :-

1. The effect of acetaminophen administration on rate of tooth movement, acid phosphatase level in serum and bone resorption was not significantly different as compared to control group.

2. The administratin of ibuprofen significantly decreased the rate of tooth movement and acid phosphatase level in serum as compared to control group and acetaminophen group.

3. The administration of nimesulide also significantly decreased the rate of tooth movement and acid phosphatase level in serum as compared to control group and acetaminophen group.

4. The effect of ibuprofen and nimesulide administration on the rate of tooth movement and acid phosphatase level in serum was not significantly different when compared with each other.

5. The effect of acetaminophen administration on the rate of bone resorption was not significantly different as compared to control group.

6. The administration of ibuprofen and nimesulide significantly decreased the rate of bone resorption and appearance of osteoclasts as compared to control group and acetaminophen group, but was not significantly different when compared with each other.

7. When the histological findings and biochemical findings were correlated with other a high correlation was found in between them.

Thus it can be concluded that acid phosphatase level in serum reflects the alveolar bone turn-over during orthodontic tooth movement.

This study suggest that acetaminophen should be the drug of choice for pain control in orthodontic practice as it has minimal adverse effect on orthodontic tooth movement.

\section{References:-}

[1]. Angle Edward H : Treatment of malocclusion of teeth. Ed. 7 Philadelphia 1907 chap 6

[2]. Baumrind Sheldon : A reconsideration of the property of the pressure and tension hypothesis. Am. J. Orthod \& Dentofac. Orthop $.55 ; 12-21: 1969$

[3]. Binderman I : The transduction of mechanical force into biochemical event in bone cells may involve activation of phospholipase A2.Calcif Tissue Int. 42; 261-6:1988

[4]. Breitnar C : Bone changes resulting from experimental orthodontics treatment. Am.J. Orthod \& Dentofac. Orthop. 26:521 : 1940

[5]. Buck D.L. \& Church D. H. : a histologic study of human tooth movement .Am>J. Orthod. \& Dentofac. Orthop. 62;507-516;1972

[6]. Burstone M. S : Histochemical demonstrationof acid phosphatase activity in osteoclasts. J. Histochem Cytochem. 7,39-41,1959

[7]. Chumbley A.B. \& Tuncay O.C. : the effects of indomethacin ( an aspirin like drug) on the rate of orthodontic tooth movement . Am.J. Orthod \& Dentofac. Orthop. 89;312-314;1986

[8]. Davidovitch Z \& Shanfeld J.L. : Biochemical mediators of the effects of mechanical forces \& electric currents on mineralized tissues. Calcif Tissue Int. 36;586-597: 1984

[9]. Davidovitch \& Shanfeld : Cyclic AMP level in alveolar bone of orthodontically treated cats.

[10]. Feldman R. S. et al : Inhibition of alveolarbone loss in humans by aspirin. J. Dent. Resc.59; Special issue A; 1980.

[11]. Golamman M \& Gianelly A : Histology of tooth movement. DCNA; 439-448 : 1972

[12]. Keeling Stephen et al : Serum \& alveolar bone phosphatase changes reflect bone turnover during orthodontic tooth movement Am.J. Orthod \& Dentofac. Orthop. 103;320-326; 1993.

[13]. Kehoe M. J. : The effect of acetaminophen, ibuprofen \& misoprostol on prostaglandin E2 synthesis \& the degree \& rate of orthodontic tooth movement. Angle Orthod. 66 ;339-350: 1996.

[14]. Kharbanda O.P \& Mohaptra P: A review of Arachidonic Acid metabolite \& Orthodontic tooth movement .JIDA: 66 ; $287-292$ : 1995

[15]. Klein D.C. \& Raisz L.G : Prostaglandin - Stimulatin of bone resorption in tissue culture . Endocrinology : 86: $1436-40: 1970$

[16]. Samuelson et al : Pathways of arachidonic acid metabolism its advances in inflammation research. New York : 1975, Raven Press Vol I, P 405

[17]. Takimoto , Kazuo Deguchi \& Mori : Histochemical detection of acid \& alkaline phosphatase in periodontal tissue after experimental tooth movement. J.Dent Res. 47; 340 ; 1968. 